\title{
Erratum: Geometric Frustration and Solid-Solid Transitions in Model 2D Tissue [Phys. Rev. Lett. 120, 268105 (2018)]
}

\author{
Michael Moshe, Mark J. Bowick, and M. Cristina Marchetti
}

(Received 22 September 2019; published 25 October 2019)

DOI: 10.1103/PhysRevLett.123.179901

We correct two errors in the calculation of the effective elastic energy formally given in Eqs. (3) and (4) of our Letter, and whose derivation is given in the Supplemental Material (SM) [1]. We also show that these two errors effectively compensate each other, so that our results are qualitatively unchanged.

First, while the expression given for the tensor $A_{P}^{\alpha \beta \gamma \delta}$ in Eq. (3) is correct, the corresponding expression for the elastic tensor for the area energy is not. This should read

$$
A_{A}^{\alpha \beta \gamma \delta}=\lambda_{A} \overline{\mathfrak{g}}_{A}^{\alpha \beta} \overline{\mathfrak{g}}_{A}^{\gamma \delta}
$$

Similarly, Eq. (22) of the SM [1] should read

$$
A_{A}^{\mu \nu \rho \sigma}=\frac{2}{3} \kappa_{A} A_{0} \overline{\mathfrak{g}}_{A}^{\mu \nu} \overline{\mathfrak{g}}_{A}^{\rho \sigma} .
$$

Indeed, within harmonic elasticity, a single elastic constant $\left(\lambda_{A}\right)$ should control the pure area changes resulting from the isotropic compression or expansion of an isotropic solid.

The second error is that an expansion of the exact continuum energy to quadratic order in the strain, given in Eq. (6) of the SM [1], is not sufficient to describe the long-wavelength limit of the cellular-tissue-vertex model. To obtain an effective long-wavelength elastic energy that captures the transition between compatible and incompatible solids one needs to incorporate higher order nonlinearities. As often happens in perturbative expansions of field theories, these higher order terms can be incorporated into an effective quadratic energy with renormalized coupling constants. This procedure yields corrections to the values of $\lambda_{P}, \mu_{P}$, and $\lambda_{A}$ and, importantly, generates a nonzero value for $\mu_{A}$, validating the form originally proposed in Eqs. (3) and (4).

The inadequacy of a purely quadratic approximation for the effective continuum energy can be understood by noting that area- and perimeter-preserving deformations are indistinguishable to linear order in the strain. To see this, consider a reference state quantified by a reference metric $\overline{\mathfrak{g}}_{i j}=\delta_{i j}$. By construction, the following deformed metrics conserve the perimeter and area of a rectangular domain

$$
\mathfrak{g}_{P}=\left(\begin{array}{cc}
(1+\delta)^{2} & 0 \\
0 & (1-\delta)^{2}
\end{array}\right), \mathfrak{g}_{A}=\left(\begin{array}{cc}
(1+\delta)^{2} & 0 \\
0 & (1+\delta)^{-2}
\end{array}\right)
$$

These two deformations are identical to linear order in $\delta$ :

$$
\mathfrak{g}_{A}-\overline{\mathfrak{g}}=\mathfrak{g}_{P}-\overline{\mathfrak{g}}=2 \delta\left(\begin{array}{cc}
1 & 0 \\
0 & -1
\end{array}\right)+O(\delta)^{2}
$$

The corresponding energies are also identical to quadratic order in the strain. It is then clear that to distinguish between areapreserving and perimeter-preserving deformations one needs to go beyond linear order in the strain in calculating the effective energy.

To validate the effective quadratic energy summarized in Eq. (6) of our Letter, we have compared it to the results obtained from the exact continuum model given in Eq. (6) of the SM [1] by calculating the Young's modulus as a function of shape index. This was done using the procedure leading to Fig. 3(a) of our Letter. For arbitrary elastic constants of order unity, the revised Fig. 3 displayed here shows that the exact and the proposed effective quadratic models exhibit a transition at the same critical shape index and display the same qualitative behavior for the Young's modulus.

The rest of the results and conclusions in our Letter are unaffected. 
PHYSICAL REVIEW LETTERS 123, 179901(E) (2019)
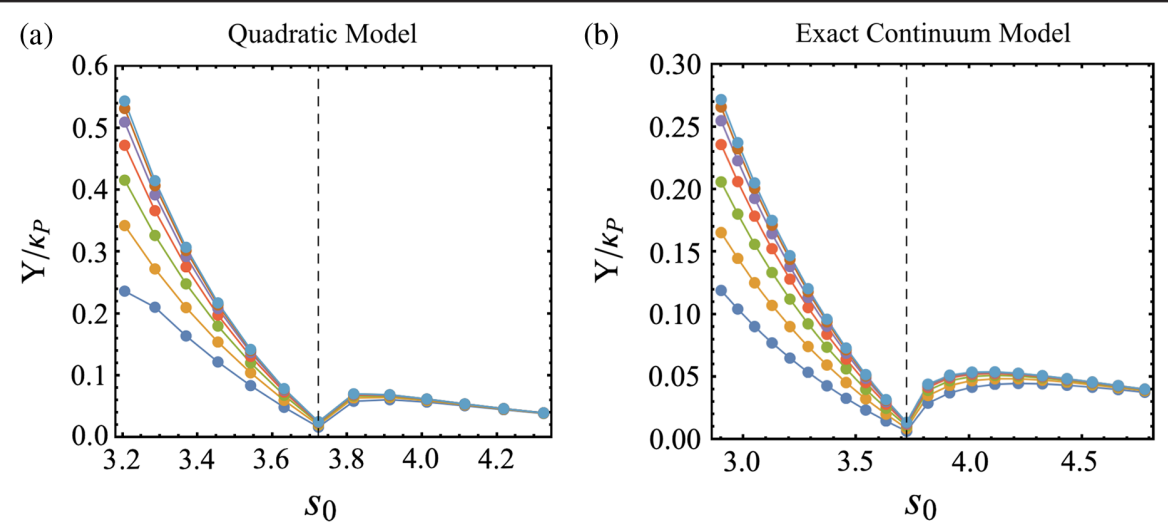

FIG. 3. Effective Young's modulus as measured by the resistance of the tissue to uniaxial strain. (a) Results based on the effective quadratic energy given in Eqs. (3), (4), and (6) of the main text, with elastic coefficients of order unity. (b) Results based on the exact continuum model as given in Eq. (6) of the Supplemental Material [1].

We thank Arturo Hernandez from the University of California at Santa-Barbara for identifying the error in calculating the area-elastic-tensor $A_{A}$.

[1] See Supplemental Material at http://link.aps.org/supplemental/10.1103/PhysRevLett.120.268105 for detailed derivation of the model, and for a Mathematica code recovering the results presented in the Letter. 